\title{
On Symplectic Analysis for the Plane Elasticity Problem of Quasicrystals with Point Group $12 \mathrm{~mm}$
}

\author{
Hua Wang, Jianrui Chen, and Xiaoyu Zhang \\ College of Sciences, Inner Mongolia University of Technology, Hohhot 010051, China \\ Correspondence should be addressed to Hua Wang; huawang96@yahoo.com
}

Received 13 March 2014; Accepted 29 May 2014; Published 23 June 2014

Academic Editor: Patricia J. Y. Wong

Copyright (C) 2014 Hua Wang et al. This is an open access article distributed under the Creative Commons Attribution License, which permits unrestricted use, distribution, and reproduction in any medium, provided the original work is properly cited.

\begin{abstract}
The symplectic approach, the separation of variables based on Hamiltonian systems, for the plane elasticity problem of quasicrystals with point group $12 \mathrm{~mm}$ is developed. By introducing appropriate transformations, the basic equations of the problem are converted to two independent Hamiltonian dual equations, and the associated Hamiltonian operator matrices are obtained. The study of the operator matrices shows the feasibility of the method. Without any assumptions, the general solution is presented for the problem with mixed boundary conditions.
\end{abstract}

\section{Introduction}

Quasicrystals (QCs), a new material and structure, were first discovered by the authors in [1] in 1984. QCs that exhibit excellent physical and mechanical properties, such as low friction, high hardness, and high wear resistance, have promising potential applications (cf. [2]). It is well known that the general solution of quasicrystal elasticity is very important, but it is difficult to be obtained because of the complexity of the basic governing equations. So far, many methods and techniques have been developed to seek for the general solution (see, e.g., [3-8]). However, some problems of quasicrystal elasticity have not been solved well due to the complicated assumptions of the solution, and the symplectic approach, developed by Zhong [9], may be helpful in those problems.

The symplectic approach has advantages of avoiding the difficulty of solving high order differential equations and having no any further assumptions and has been applied into various research fields such as elasticity [10-12], viscoelasticity [13], fluid mechanics [14], piezoelectric material [15], and functionally graded effects [16]. In this method, one needs to transform the considered problem into Hamiltonian dual equations and then obtains the desired Hamiltonian operator matrix. Based on the eigenvalue analysis and eigenfunction expansion, the analytical solution of the problem can be explicitly presented. It should be noted that the feasibility of this method depends on the completeness of eigenfunction systems of the corresponding Hamiltonian operator matrices.

To the best of the author's knowledge, there are no reports of the method on the analysis of QCs. The objective of this paper is to propose the symplectic approach for the plane elasticity problem of quasicrystals with point group $12 \mathrm{~mm}$. After derivation of two independent Hamiltonian dual equations of the problem, we prove the completeness of eigenfunction systems for the corresponding Hamiltonian operator matrices. Finally, we obtain the analytical solution with the use of the eigenfunction expansion.

\section{Basic Equations and Their Hamiltonian Dual Equations}

According to the quasicrystal elasticity theory, we have the deformation geometry equations of the plane elasticity problem of quasicrystals with point group $12 \mathrm{~mm}$

$$
\varepsilon_{i j}=\frac{1}{2}\left(\frac{\partial u_{i}}{\partial x_{j}}+\frac{\partial u_{j}}{\partial x_{i}}\right), \quad w_{i j}=\frac{\partial w_{i}}{\partial x_{j}}, \quad i, j=1,2
$$


the equilibrium equations

$$
\frac{\partial \sigma_{i j}}{\partial x_{j}}+f_{i}=0, \quad \frac{\partial H_{i j}}{\partial x_{j}}+g_{i}=0,
$$

and the generalized Hooke's law

$$
\begin{aligned}
& \sigma_{x x}=C_{12}\left(\varepsilon_{x x}+\varepsilon_{y y}\right)+2 C_{66} \varepsilon_{x x}, \\
& \sigma_{y y}=C_{12}\left(\varepsilon_{x x}+\varepsilon_{y y}\right)+2 C_{66} \varepsilon_{y y}, \\
& \sigma_{x y}=\sigma_{y x}=2 C_{66} \varepsilon_{x y}, \\
& H_{x x}=K_{1} w_{x x}+K_{2} w_{y y}, \\
& H_{y y}=K_{1} w_{y y}+K_{2} w_{x x}, \\
& H_{x y}=\left(K_{1}+K_{2}+K_{3}\right) w_{x y}+K_{3} w_{y x}, \\
& H_{y x}=\left(K_{1}+K_{2}+K_{3}\right) w_{y x}+K_{3} w_{x y} .
\end{aligned}
$$

Here $u_{i}$ and $w_{i}$ are the phonon and phason displacements, $\sigma_{i j}$ and $\varepsilon_{i j}$ are the phonon stresses and strains, $H_{i j}$ and $w_{i j}$ are the phason stresses and strains, $C_{12}, C_{66}, K_{1}, K_{2}$, and $K_{3}$ are the elastic constants, and $f_{i}$ and $g_{i}$ are the body and generalized body forces, respectively.

Substituting (1) and (3) into (2), we get the displacement equilibrium equations

$$
\begin{gathered}
C_{66} \nabla^{2} u_{x}+\left(C_{12}+C_{66}\right) \frac{\partial}{\partial x}\left(\frac{\partial u_{x}}{\partial x}+\frac{\partial u_{y}}{\partial y}\right)+f_{1}=0 \\
C_{66} \nabla^{2} u_{y}+\left(C_{12}+C_{66}\right) \frac{\partial}{\partial y}\left(\frac{\partial u_{x}}{\partial x}+\frac{\partial u_{y}}{\partial y}\right)+f_{2}=0, \\
K_{1} \nabla^{2} w_{x}+\left(K_{2}+K_{3}\right) \frac{\partial}{\partial y}\left(\frac{\partial w_{x}}{\partial y}+\frac{\partial w_{y}}{\partial x}\right)+g_{1}=0, \\
K_{1} \nabla^{2} w_{y}+\left(K_{2}+K_{3}\right) \frac{\partial}{\partial x}\left(\frac{\partial w_{x}}{\partial y}+\frac{\partial w_{y}}{\partial x}\right)+g_{2}=0
\end{gathered}
$$

where $\nabla^{2}=\left(\partial^{2} / \partial x^{2}\right)+\left(\partial^{2} / \partial y^{2}\right)$. Let

$$
\begin{aligned}
& q_{1}=\frac{C_{66}}{C_{12}+2 C_{66}}\left(\frac{\partial u_{x}}{\partial y}-\frac{\partial u_{y}}{\partial x}\right), \\
& q_{2}=\frac{\partial u_{x}}{\partial x}+\frac{\partial u_{y}}{\partial y}, \\
& q_{3}=\frac{K_{1}+K_{2}+K_{3}}{K_{1}}\left(\frac{\partial w_{x}}{\partial y}+\frac{\partial w_{y}}{\partial x}\right), \\
& q_{4}=-\frac{\partial w_{x}}{\partial x}+\frac{\partial w_{y}}{\partial y} .
\end{aligned}
$$

Then (4) can be expressed in the following matrix forms:

$$
\begin{aligned}
& \frac{\partial}{\partial y} Z_{1}=H_{1} Z_{1}+F_{1}, \\
& \frac{\partial}{\partial y} Z_{2}=H_{2} Z_{2}+F_{2},
\end{aligned}
$$

where $Z_{1}=\left(u_{x}, u_{y}, q_{1}, q_{2}\right)^{T}, Z_{2}=\left(w_{x}, w_{y}, q_{3}, q_{4}\right)^{T}, F_{1}=$ $-\left(1 /\left(C_{12}+2 C_{66}\right)\right)\left(0,0, f_{1}, f_{2}\right)^{T}, F_{2}=\left(-1 / K_{1}\right)\left(0,0, g_{1}, g_{2}\right)^{T}$,

$$
\begin{aligned}
& H_{1}=\left(\begin{array}{cccc}
0 & \frac{\partial}{\partial x} & \frac{C_{12}+2 C_{66}}{C_{66}} & 0 \\
-\frac{\partial}{\partial x} & 0 & 0 & 1 \\
0 & 0 & 0 & -\frac{\partial}{\partial x} \\
0 & 0 & \frac{\partial}{\partial x} & 0
\end{array}\right), \\
& H_{2}=\left(\begin{array}{cccc}
0 & -\frac{\partial}{\partial x} & \frac{K_{1}}{K_{1}+K_{2}+K_{3}} & 0 \\
\frac{\partial}{\partial x} & 0 & 0 & 1 \\
0 & 0 & 0 & \frac{\partial}{\partial x} \\
0 & 0 & -\frac{\partial}{\partial x} & 0
\end{array}\right) .
\end{aligned}
$$

Obviously, $H_{1}^{T}=J H_{1} J$ and $H_{2}^{T}=J H_{2} J$, in which $J=\left(\begin{array}{cc}0 & I_{2} \\ -I_{2} & 0\end{array}\right)$ is the symplectic matrix with $I_{2}$ being the $2 \times 2$ identity matrix. Thus, $H_{1}$ and $H_{2}$ are both Hamiltonian operator matrices and (6) and (7) are exactly the Hamiltonian dual equations for the plane elasticity problem of quasicrystals with point group $12 \mathrm{~mm}$.

We consider the problem satisfying the mixed boundary conditions

$$
\begin{aligned}
& u_{x}=0, \quad \sigma_{x y}=0, \quad \text { for } x=0, x=h, \\
& w_{x}=0, \quad H_{x y}=0, \quad \text { for } x=0, x=h .
\end{aligned}
$$

From (9) and (3), we have

$$
q_{1}=q_{3}=0, \quad \text { for } x=0, x=h .
$$

\section{Theoretical Analysis}

In the following, we only discuss (6), and the analysis for (7) is similar.

First, considering the homogeneous equation of (6),

$$
\frac{\partial}{\partial y} Z_{1}=H_{1} Z_{1}
$$

Applying the method of separation of variables to the above equation, we write the solution as

$$
Z_{1}=X(x) Y(y),
$$

in which $Y(y)=e^{\mu y}$, and $\mu$ and $X(x)$ are the eigenvalue of the Hamiltonian operator matrix $H_{1}$ and its associated eigenvector, respectively. They are determined by the equation

$$
H_{1} X(x)=\mu X(x) \text {. }
$$

Solving (13) with the boundary conditions (9) and (10) at $x=0, h$, we obtain the eigenvalues of $H_{1}$ :

$$
\begin{array}{r}
\mu_{0}=0, \quad \mu_{n}=\frac{n \pi}{h}, \quad \mu_{-n}=-\frac{n \pi}{h}, \\
n=1,2, \ldots,
\end{array}
$$


and the associated eigenvectors of $\mu_{0}, \mu_{n}$, and $\mu_{-n}$ are

$$
\begin{gathered}
X_{0}^{0}=\left(\begin{array}{l}
0 \\
1 \\
0 \\
0
\end{array}\right), \quad X_{n}^{0}=\left(\begin{array}{c}
\sin \left(\mu_{n} x\right) \\
-\cos \left(\mu_{n} x\right) \\
0 \\
0
\end{array}\right), \\
X_{-n}^{0}=\left(\begin{array}{c}
-\sin \left(\mu_{n} x\right) \\
-\cos \left(\mu_{n} x\right) \\
0 \\
0
\end{array}\right),
\end{gathered}
$$

respectively. From

$$
H_{1} X_{n}^{1}(x)=\mu_{n} X_{n}^{1}(x)+X_{n}^{0}(x)
$$

and the imposed boundary conditions, the first-order Jordan form eigenvectors of $\mu_{0}, \mu_{n}$, and $\mu_{-n}$ can be solved as

$$
X_{n}^{1}=\left(\begin{array}{c}
X_{0}^{1}=\left(\begin{array}{c}
0 \\
0 \\
0 \\
1
\end{array}\right), \\
\sin \left(\mu_{n} x\right) \\
\left(\frac{C_{12}+3 C_{66}}{\left(C_{12}+C_{66}\right) \mu_{n}}-1\right) \cos \left(\mu_{n} x\right) \\
\frac{2 C_{66}}{C_{12}+C_{66}} \sin \left(\mu_{n} x\right) \\
\frac{2 C_{66}}{C_{12}+C_{66}} \cos \left(\mu_{n} x\right) \\
-\sin \left(\mu_{n} x\right) \\
-\left(\frac{C_{12}+3 C_{66}}{\left(C_{12}+C_{66}\right) \mu_{n}}+1\right) \cos \left(\mu_{n} x\right) \\
\frac{2 C_{66}}{C_{12}+C_{66}} \sin \left(\mu_{n} x\right) \\
\frac{2 C_{66}}{C_{12}+C_{66}} \cos \left(\mu_{n} x\right)
\end{array}\right),
$$

respectively. Besides, we can verify that there are no other high-order Jordan form eigenvectors in every chain.

It is easy to prove that the above eigenvectors and Jordan form eigenvectors satisfy the symplectic conjugacy and orthogonality; that is,

$$
\begin{aligned}
\int_{0}^{h} X_{0}^{0^{T}} J X_{0}^{0} d x & =\int_{0}^{h} X_{0}^{0^{T}} J X_{n}^{1} d x=\int_{0}^{h} X_{0}^{1^{T}} J X_{n}^{0} d x \\
& =\int_{0}^{h} X_{0}^{1^{T}} J X_{n}^{1} d x=0,
\end{aligned}
$$

$$
\begin{aligned}
\int_{0}^{h} X_{n}^{0^{T}} J X_{n}^{0} d x & =\int_{0}^{h} X_{n}^{0^{T}} J X_{n}^{1} d x=\int_{0}^{h} X_{n}^{0^{T}} J X_{-n}^{0} d x \\
& =\int_{0}^{h} X_{n}^{1^{T}} J X_{-n}^{1} d x=0, \\
\int_{0}^{h} X_{0}^{0^{T}} J X_{0}^{1} d x=h, \quad \int_{0}^{h} X_{n}^{0^{T}} J X_{-n}^{1} d x & =-\frac{2 C_{66}}{C_{12}+C_{66}} h, \\
n & = \pm 1, \pm 2, \ldots
\end{aligned}
$$

Next, we will prove the symplectic orthogonal expansion theorem, that is, the completeness theorem of the generalized eigenvector system (i.e., the collection of all the eigenvectors and Jordan form eigenvectors), which shows that the symplectic method can be adopted to solve the title problem.

Theorem 1. The generalized eigenvector system

$$
\left\{X_{0}^{0}, X_{0}^{1}\right\} \cup\left\{X_{n}^{0}, X_{n}^{1} \mid n= \pm 1, \pm 2, \ldots\right\}
$$

of the Hamiltonian operator matrix $H_{1}$ is complete in the Hilbert space $X$; that is, there exist constant sequences $\left\{c_{0}^{0}, c_{0}^{1}\right\}$, $\left\{c_{n}^{i}\right\}_{n=1}^{\infty}$, and $\left\{c_{-n}^{i}\right\}_{n=1}^{\infty}(i=0,1)$ such that

$$
\Phi=c_{0}^{0} X_{0}^{0}+c_{0}^{1} X_{0}^{1}+\sum_{n=1}^{+\infty}\left(c_{n}^{0} X_{n}^{0}+c_{n}^{1} X_{n}^{1}+c_{-n}^{0} X_{-n}^{0}+c_{-n}^{1} X_{-n}^{1}\right)
$$

for each $\Phi=\left(\phi_{1}(x), \phi_{2}(x), \phi_{3}(x), \phi_{4}(x)\right)^{T} \in X$, where $X=$ $L^{2}[0, h] \times L^{2}[0, h] \times L^{2}[0, h] \times L^{2}[0, h]$.

Proof. For any $\Phi \in V$, in order to prove equality (20), we set

$$
\begin{aligned}
& c_{0}^{0}=\frac{\int_{0}^{h} \Phi^{T} J X_{0}^{1} d x}{\int_{0}^{h} X_{0}^{0^{T}} J X_{0}^{1} d x}=\frac{\int_{0}^{h} \phi_{2} d x}{h}, \\
& c_{0}^{1}=\frac{\int_{0}^{h} \Phi^{T} J X_{0}^{0} d x}{\int_{0}^{h} X_{0}^{1^{T}} J X_{0}^{0} d x}=\frac{\int_{0}^{h} \phi_{4} d x}{h},
\end{aligned}
$$

$$
\begin{aligned}
c_{n}^{0}= & \frac{\int_{0}^{h} \Phi^{T} J X_{-n}^{1} d x}{\int_{0}^{h} X_{n}^{0^{T}} J X_{-n}^{1} d x} \\
= & \frac{\int_{0}^{h} \phi_{1} \sin \left(\mu_{n} x\right) d x-\int_{0}^{h} \phi_{2} \cos \left(\mu_{n} x\right) d x}{h} \\
& -\left(\int_{0}^{h} \phi_{3} \sin \left(\mu_{n} x\right) d y+\left(\frac{\left(C_{12}+3 C_{66}\right)}{\left(C_{12}+C_{66}\right) \mu_{n}}+1\right)\right. \\
& \left.\quad \times \int_{0}^{h} \phi_{4} \cos \left(\mu_{n} x\right) d x\right) \times\left(\frac{2 C_{66}}{C_{12}+C_{66}} h\right)^{-1},
\end{aligned}
$$




$$
\begin{aligned}
c_{n}^{1} & =\frac{\int_{0}^{h} \Phi^{T} J X_{-n}^{0} d x}{\int_{0}^{h} X_{n}^{1^{T}} J X_{-n}^{0} d x} \\
& =\frac{\int_{0}^{h} \phi_{3} \sin \left(\mu_{n} x\right) d x+\int_{0}^{h} \phi_{4} \cos \left(\mu_{n} x\right) d x}{\left(2 C_{66} /\left(C_{12}+C_{66}\right)\right) h}
\end{aligned}
$$

by the symplectic orthogonal relationship (18). Then,

$$
\begin{gathered}
c_{0}^{0} X_{0}^{0}+c_{0}^{1} X_{0}^{1}+\sum_{n=1}^{+\infty}\left(c_{n}^{0} X_{n}^{0}+c_{n}^{1} X_{n}^{1}+c_{-n}^{0} X_{-n}^{0}+c_{-n}^{1} X_{-n}^{1}\right) \\
=\left(\begin{array}{c}
\sum_{n=1}^{+\infty} \frac{2}{h} \int_{0}^{h}\left(\phi_{1} \sin \frac{n \pi x}{h} d x\right) \sin \frac{n \pi x}{h} \\
\frac{1}{h} \int_{0}^{h} \phi_{2} d x+\sum_{n=1}^{+\infty} \frac{2}{h}\left(\int_{0}^{h} \phi_{2} \cos \frac{n \pi x}{h} d x\right) \cos \frac{n \pi x}{h} \\
\sum_{n=1}^{+\infty} \frac{2}{h} \int_{0}^{1}\left(\phi_{3} \sin \frac{n \pi x}{h} d x\right) \sin \frac{n \pi x}{h} \\
\int_{0}^{h} \phi_{4} d x+\sum_{n=1}^{+\infty} \frac{2}{h}\left(\int_{0}^{h} \phi_{4} \cos \frac{n \pi x}{h} d x\right) \cos \frac{n \pi x}{h}
\end{array}\right),
\end{gathered}
$$

in which the four components of the above expression are the corresponding Fourier series of $\phi_{1}, \phi_{2}, \phi_{3}, \phi_{4}$ associated with the orthogonal function system $\{\sin (n \pi x / h)\}_{n=1}^{+\infty}$ or $\{\cos (n \pi x / h)\}_{n=0}^{+\infty}$ in $L^{2}[0, h]$. Therefore, equality (20) is valid, which means that the generalized eigenvector system of $H_{1}$ is complete in the Hilbert space $X$.

Similarly, for the Hamiltonian operator matrix $\mathrm{H}_{2}$, we also have the following completeness theory.

Theorem 2. The generalized eigenvector system

$$
\left\{\bar{X}_{0}^{0}, \bar{X}_{0}^{1}\right\} \cup\left\{\bar{X}_{n}^{0}, \bar{X}_{n}^{1} \mid n= \pm 1, \pm 2, \ldots\right\}
$$

of the Hamiltonian operator matrix $\mathrm{H}_{2}$ is complete in the Hilbert space $X$, where

$$
\begin{gathered}
\bar{X}_{0}^{0}=\left(\begin{array}{l}
0 \\
1 \\
0 \\
0
\end{array}\right), \quad \bar{X}_{n}^{0}=\left(\begin{array}{c}
\sin \left(\lambda_{n} x\right) \\
\cos \left(\lambda_{n} x\right) \\
0 \\
0
\end{array}\right), \\
\bar{X}_{0}^{1}=\left(\begin{array}{l}
0 \\
0 \\
0 \\
1
\end{array}\right), \\
\bar{X}_{n}^{1}=\left(\begin{array}{c}
\left(\begin{array}{c}
2 K_{1}+K_{2}+K_{3} \\
\left.1+\frac{\lambda_{n}\left(K_{2}+K_{3}\right)}{2}\right) \cos \left(\lambda_{n} x\right) \\
-\left(\frac{2 K_{1}}{K_{2}+K_{3}}+2\right) \sin \left(\lambda_{n} x\right) \\
\left(\frac{2 K_{1}}{K_{2}+K_{3}}+2\right) \cos \left(\lambda_{n} x\right)
\end{array}\right)
\end{array}\right.
\end{gathered}
$$

are the associated eigenvectors and the first-order Jordan form eigenvectors of the eigenvalue $\lambda_{0}=0$ and $\lambda_{n}=n \pi / h$ of $\mathrm{H}_{2}$.

\section{General Solution}

By completeness Theorem 1, the general solution of the inhomogeneous equation (6) is represented in the form

$$
\begin{aligned}
& Z_{1}(x, y)= X_{0}^{0} Y_{0}^{0}(y)+X_{0}^{1} Y_{0}^{1}(y) \\
&+\sum_{n=1}^{+\infty}\left(X_{n}^{0} Y_{n}^{0}(y)+X_{n}^{1} Y_{n}^{1}(y)+X_{-n}^{0} Y_{-n}^{0}(y)\right. \\
&\left.\quad+X_{-n}^{1} Y_{-n}^{1}(y)\right) .
\end{aligned}
$$

The vector $F_{1}$ can also be expanded as

$$
\begin{aligned}
F_{1}= & X_{0}^{0} F_{0}^{0}(y)+X_{0}^{1} F_{0}^{1}(y) \\
+\sum_{n=1}^{+\infty}( & X_{n}^{0} F_{n}^{0}(y)+X_{n}^{1} F_{n}^{1}(y)+X_{-n}^{0} F_{-n}^{0}(y) \\
& \left.+X_{-n}^{1} F_{-n}^{1}(y)\right) .
\end{aligned}
$$

Multiplying both sides of (26) by $X_{0}^{1^{T}} J, X_{0}^{0^{T}} J, X_{-n}^{1}{ }^{T} J, X_{-n}^{0}{ }^{T} J$, $X_{n}^{1^{T}} J$, and $X_{n}^{0^{T}} J$ and then integrating by $x$ from 0 to $h$, respectively, we have

$$
F_{0}^{0}(y)=\frac{\int_{0}^{h} X_{0}^{1^{T}} J F_{1} d x}{\int_{0}^{h} X_{0}^{1^{T}} J X_{0}^{0} d x}=0
$$

$$
\begin{aligned}
F_{0}^{1}(y)= & \frac{\int_{0}^{h} X_{0}^{0^{T}} J F_{1} d x}{\int_{0}^{h} X_{0}^{0^{T}} J X_{0}^{1} d x}=-\frac{\int_{0}^{h} f_{2} d x}{\left(C_{12}+2 C_{66}\right) h}, \\
F_{n}^{0}(y)= & \frac{\int_{0}^{h} X_{-n}^{1}{ }^{T} J F_{1} d x}{\int_{0}^{h} X_{-n}^{1} J X_{n}^{0} d x} \\
= & \left(\int_{0}^{h} f_{1} \sin \left(\mu_{n} x\right) d x+\left(\frac{C_{12}+3 C_{66}}{\left(C_{12}+C_{66}\right) \mu_{n}}+1\right)\right. \\
& \left.\times \int_{0}^{h} f_{2} \cos \left(\mu_{n} x\right) d x\right) \\
& \times\left(\frac{2 C_{66}\left(C_{12}+2 C_{66}\right)}{C_{12}+C_{66}} h\right)^{-1}, \\
F_{n}^{1}(y)= & \frac{\int_{0}^{h} X_{-n}^{0} J F_{1} d x}{\int_{0}^{h} X_{-n}^{0} J X_{n}^{1} d x} \\
= & \frac{-\int_{0}^{h} f_{1} \sin \left(\mu_{n} x\right) d x-\int_{0}^{h} f_{2} \cos \left(\mu_{n} x\right) d x}{\left(2 C_{66}\left(C_{12}+2 C_{66}\right) /\left(C_{12}+C_{66}\right)\right) h} \\
n= \pm 1, \pm 2, \ldots & n=127)
\end{aligned}
$$


TABLE 1: The computed results.

\begin{tabular}{lccccc}
\hline$(x, y)$ & $(0.1464,0.8536)$ & $(1.4,0.55)$ & $(2.1,0.34)$ & $(3.42,0.89)$ & $(4.7,0.27)$ \\
\hline$u_{x}$ & 0.204 & -0.08131 & -0.237 & 0.4822 & -0.2427 \\
$u_{y}$ & -0.06272 & -0.01618 & -0.02746 & -0.0399 & -0.04801 \\
$w_{x}$ & 0.2965 & -0.243 & -0.583 & 0.6355 & -0.4975 \\
$w_{y}$ & -0.02621 & -0.006761 & -0.01148 & -0.01667 & -0.02006 \\
\hline
\end{tabular}

Then, substituting (25) and (26) into (6) yields

$$
\begin{gathered}
\frac{d Y_{0}^{1}(y)}{d y}=F_{0}^{1}(y), \quad \frac{d Y_{0}^{0}(y)}{d y}=Y_{0}^{1}(y), \\
\frac{d Y_{n}^{1}(y)}{d y}=\mu_{n} Y_{n}^{1}(y)+F_{n}^{1}(y), \\
\frac{d Y_{n}^{0}(y)}{d y}=\mu_{n} Y_{n}^{0}(y)+Y_{n}^{1}(y)+F_{n}^{0}(y) .
\end{gathered}
$$

Thus, we obtain

$$
\begin{gathered}
Y_{0}^{1}(y)=c_{0}^{1}+\int_{0}^{y} F_{0}^{1}(\xi) d \xi \\
Y_{0}^{0}(y)=c_{0}^{0}+c_{0}^{1} y+\int_{0}^{y} \int_{0}^{\tau} F_{0}^{1}(\xi) d \xi d \tau, \\
Y_{n}^{1}(y)=c_{n}^{1} e^{\mu_{n} y}+\int_{0}^{y} F_{n}^{1}(\xi) e^{\mu_{n}(y-\xi)} d \xi \\
Y_{n}^{0}(y)=\left(c_{n}^{0}+c_{n}^{1} y\right) e^{\mu_{n} y}+\int_{0}^{y} F_{n}^{0}(\xi) e^{\mu_{n}(y-\xi)} d \xi \\
+\int_{0}^{y} \int_{0}^{\tau} F_{n}^{1}(\xi) e^{\mu_{n}(y-\xi)} d \xi d \tau,
\end{gathered}
$$

where $c_{0}^{1}, c_{0}^{0}, c_{n}^{1}$, and $c_{n}^{0}$ are unknown constants to be determined by imposing the remaining boundary conditions at $y$. Substituting (29) into (25), we have the analytical solutions $u_{x}$ and $u_{y}$ of (4) given by

$$
\begin{aligned}
& u_{x} \\
& =\sum_{n=1}^{+\infty}\left[\left(c_{n}^{0}+c_{n}^{1}+c_{n}^{1} y\right) e^{\mu_{n} y}\right. \\
& \quad-\left(c_{-n}^{0}+c_{-n}^{1}+c_{-n}^{1} y\right) e^{-\mu_{n} y} \\
& +\int_{0}^{y}\left(\left(F_{n}^{0}(\xi)+F_{n}^{1}(\xi)\right) e^{\mu_{n}(y-\xi)}\right. \\
& \quad+\int_{0}^{y} \int_{0}^{\tau}\left(F_{n}^{1}(\xi) e^{\mu_{n}(y-\xi)}\right. \\
& \left.\left.-F_{-n}^{1}(\xi) e^{-\mu_{n}(y-\xi)}\right) d \xi d \tau\right] \sin \mu_{n} x
\end{aligned}
$$

$u_{y}$

$$
\begin{gathered}
=c_{0}^{0}+c_{0}^{1} y+\int_{0}^{y} \int_{0}^{\tau} F_{0}^{1}(\xi) d \xi d \tau \\
-\sum_{n=1}^{+\infty}\left[\left(c_{n}^{0}-\left(\frac{C_{12}+3 C_{66}}{\left(C_{12}+C_{66}\right) \mu_{n}}-1\right) c_{n}^{1}+c_{n}^{1} y\right) e^{\mu_{n} y}\right. \\
+\left(c_{-n}^{0}+\left(\frac{C_{12}+3 C_{66}}{\left(C_{12}+C_{66}\right) \mu_{n}}+1\right) c_{-n}^{1}+c_{-n}^{1} y\right) e^{-\mu_{n} y} \\
+\int_{0}^{y}\left(\left(F_{n}^{0}(\xi)-\left(\frac{C_{12}+3 C_{66}}{\left(C_{12}+C_{66}\right) \mu_{n}}-1\right)\right.\right. \\
\left.\quad \times F_{n}^{1}(\xi)\right) e^{\mu_{n}(y-\xi)} \\
+\left(F_{-n}^{0}(\xi)+\left(\frac{C_{12}+3 C_{66}}{\left(C_{12}+C_{66}\right) \mu_{n}}+1\right)\right. \\
\left.+\int_{0}^{y} \int_{0}^{\tau}\left(F_{n}^{1}(\xi) e^{\mu_{n}(y-\xi)}+F_{-n}^{1}(\xi) e^{-\mu_{n}(y-\xi)}\right) d \xi d \tau\right] \\
\times \cos \mu_{n} x . \\
\left.\left.\times F_{-n}^{1}(\xi)\right) e^{-\mu_{n}(y-\xi)}\right) d \xi
\end{gathered}
$$

According to the above procedure for (7), the analytical solutions $w_{x}$ and $w_{y}$ of (4) can be obtained:

$$
\begin{aligned}
w_{x}=\sum_{n=1}^{+\infty}[ & \left(d_{n}^{0}+d_{n}^{1}+d_{n}^{1} y\right) e^{\mu_{n} y} \\
& -\left(d_{-n}^{0}+d_{-n}^{1}+d_{-n}^{1} y\right) e^{-\lambda_{n} y} \\
& +\int_{0}^{y}\left(\left(\bar{F}_{n}^{0}(\xi)+\bar{F}_{n}^{1}(\xi)\right) e^{\lambda_{n}(y-\xi)}\right. \\
& +\int_{0}^{y} \int_{0}^{\tau}\left(\bar{F}_{n}^{1}(\xi) e^{\lambda_{n}(y-\xi)}\right. \\
& \left.\left.-\bar{F}_{-n}^{1}(\xi) e^{-\lambda_{n}(y-\xi)}\right) d \xi d \tau\right] \sin \lambda_{n} x
\end{aligned}
$$




$$
\begin{aligned}
& w_{y} \\
& =d_{0}^{0}+d_{0}^{1} y+\int_{0}^{y} \int_{0}^{\tau} \bar{F}_{0}^{1}(\xi) d \xi d \tau \\
& +\sum_{n=1}^{+\infty}\left[\left(d_{n}^{0}+\left(1+\frac{2 K_{1}+K_{2}+K_{3}}{\lambda_{n}\left(K_{2}+K_{3}\right)}\right) d_{n}^{1}+d_{n}^{1} y\right) e^{\lambda_{n} y}\right. \\
& +\left(d_{-n}^{0}+\left(1-\frac{2 K_{1}+K_{2}+K_{3}}{\lambda_{n}\left(K_{2}+K_{3}\right)}\right) d_{-n}^{1}\right. \\
& \left.+d_{-n}^{1} y\right) e^{-\lambda_{n} y} \\
& +\int_{0}^{y}\left(\left(\bar{F}_{n}^{0}(\xi)+\left(1+\frac{2 K_{1}+K_{2}+K_{3}}{\lambda_{n}\left(K_{2}+K_{3}\right)}\right) \bar{F}_{n}^{1}(\xi)\right)\right. \\
& \times e^{\lambda_{n}(y-\xi)} \\
& +\left(\bar{F}_{-n}^{0}(\xi)+\left(1-\frac{2 K_{1}+K_{2}+K_{3}}{\lambda_{n}\left(K_{2}+K_{3}\right)}\right) \bar{F}_{-n}^{1}(\xi)\right) \\
& \left.\times e^{-\lambda_{n}(y-\xi)}\right) d \xi \\
& +\int_{0}^{y} \int_{0}^{\tau}\left(\bar{F}_{n}^{1}(\xi) e^{\lambda_{n}(y-\xi)}\right. \\
& \left.\left.+\bar{F}_{-n}^{1}(\xi) e^{-\lambda_{n}(y-\xi)}\right) d \xi d \tau\right] \cos \lambda_{n} x,
\end{aligned}
$$

where $d_{0}^{1}, d_{0}^{0}, d_{n}^{1}$, and $d_{n}^{0}$ are unknown constants to be determined by imposing the remaining boundary conditions at $y$ and

$$
\begin{gathered}
\bar{F}_{0}^{1}=\frac{\int_{0}^{h} g_{2} d x}{K_{1} h}, \\
\bar{F}_{n}^{1}=\frac{-\int_{0}^{h} g_{1} \sin \lambda_{n} x d x+\int_{0}^{h} g_{2} \cos \lambda_{n} x d x}{\left(\left(2 K_{1}+K_{2}+K_{3}\right) /\left(K_{2}+K_{3}\right)\right) h}, \\
\bar{F}_{n}^{0}=\left(\int_{0}^{h} g_{1} \sin \lambda_{n} x d y\right. \\
\left.-\left(1-\frac{2 K_{1}+K_{2}+K_{3}}{\lambda_{n}\left(K_{2}+K_{3}\right)}\right) \int_{0}^{h} g_{2} \cos \lambda_{n} x d x\right) \\
\times\left(\frac{2 K_{1}+K_{2}+K_{3}}{K_{2}+K_{3}} h\right)^{-1} \cdot
\end{gathered}
$$

\section{Numerical Calculations}

Compared with [17], the present paper is devoted to the symplectic analysis of the plane elasticity problem of quasicrystals. To guarantee the feasibility of our method, we also prove the completeness for the eigenfunction system of the associated Hamiltonian operator matrices. Note that the completeness does not always hold for the Hamiltonian operator matrices.
In order to determine the unknown constants $c_{n}^{k}$ and $d_{n}^{k}$ of the analytical solution in (30) and (31), we consider the boundary conditions at $y=0, y=l$ given by

$$
\begin{array}{ll}
u_{x}=\sin \frac{4 \pi}{h} x, & u_{y}=0, \quad \text { for } y=0, y=l, \\
w_{x}=\sin \frac{4 \pi}{h} x, & w_{y}=0, \quad \text { for } y=0, y=l .
\end{array}
$$

In the following, let $h=5, l=1$, and we take the constants $C_{12}=0.5714, C_{66}=0.88445, K_{1}=1.22, K_{2}=0.24$, and $K_{3}=$ 0.6 . The computed results are listed in Table 1 for illustrating previous main results, and the data is the same as that of using the treatment in [17].

\section{Conclusions}

The symplectic approach is established for the plane elasticity problem of quasicrystals with point group $12 \mathrm{~mm}$ satisfying the mixed boundary conditions. The corresponding Hamiltonian operator matrix plays an important role in this method, whose eigenvalues and eigenfunctions need to be obtained. Through calculations, the eigenfunction system is symplectic orthogonal. Based on this, we further verify the feasibility of this approach. Then the exact analytical solution is given with the use of the symplectic eigenfunction method. We can know that the method is totally rational and gives us a systematic way to solve physical problems. In addition, this approach is expected to apply to other quasicrystal problems.

\section{Conflict of Interests}

The authors declare that there is no conflict of interests regarding the publication of this paper.

\section{Acknowledgments}

The authors are grateful to the referees for valuable comments on improving this paper. This work is supported by the National Natural Science Foundation of China (nos. 11261034 and 11326239).

\section{References}

[1] D. Shechtman, I. Blech, D. Gratias, and J. W. Cahn, "Metallic phase with long-range orientational order and no translational symmetry," Physical Review Letters, vol. 53, no. 20, pp. 1951-1953, 1984.

[2] J. M. Dubois, S. S. Kang, and J. von Stebut, "Quasicrystalline low-friction coatings," Journal of Materials Science Letters, vol. 10, no. 9, pp. 537-541, 1991.

[3] T. Fan, Mathematical Theory of Elasticity of Quasicrystals and Its Applications, Springer, Berlin, Germany, 2011.

[4] D. Ding, R. Wang, W. Yang, and C. Hu, "General expressions for the elastic displacement fields induced by dislocations in quasicrystals," Journal of Physics: Condensed Matter, vol. 7, pp. 5423-5436, 1995.

[5] Y. Gao and B.-S. Zhao, "General solutions of three-dimensional problems for two-dimensional quasicrystals," Applied Mathematical Modelling, vol. 33, no. 8, pp. 3382-3391, 2009. 
[6] H. C. Yaslan, "Equations of anisotropic elastodynamics in 3D quasicrystals as a symmetric hyperbolic system: deriving the time-dependent fundamental solutions," Applied Mathematical Modelling, vol. 37, no. 18-19, pp. 8409-8418, 2013.

[7] L. H. Li and T. Y. Fan, "Exact solutions of two semi-infinite collinear cracks in a strip of one dimensional hexagonal quasicrystal," Applied Mathematics and Computation, vol. 196, no. 1, pp. 1-5, 2008.

[8] G. Liu and T. Fan, "Complex method of the plane elasticity in $2 \mathrm{D}$ quasicrystal with point group $10 \mathrm{~mm}$ tenfold rotational symmetry and holey problems," Science in China E: Technological Sciences, vol. 46, no. 3, 2003.

[9] W. Zhong, "Method of separation of variables and hamiltonian system," Computational Structural Mechanics and Applications, vol. 8, pp. 229-239, 1991.

[10] W. Zhong, A New Systematic Methodology for Theory of Elasticity, Dalian University of Technology Press, Dalian, China, 1995 (Chinese).

[11] C. W. Lim, C. F. Lü, Y. Xiang, and W. Yao, “On new symplectic elasticity approach for exact free vibration solutions of rectangular Kirchhoff plates," International Journal of Engineering Science, vol. 47, no. 1, pp. 131-140, 2009.

[12] B. Pan, R. Li, Y. Su, B. Wang, and Y. Zhong, "Analytical bending solutions of clamped rectangular thin plates resting on elastic foundations by the symplectic superposition method," Applied Mathematics Letters, vol. 26, no. 3, pp. 355-361, 2013.

[13] X. Xu, W. Zhang, X. Li, and G. Wang, "An application of the symplectic system in two-dimensional viscoelasticity," International Journal of Engineering Science, vol. 44, no. 13-14, pp. 897914, 2006.

[14] G. P. Wang, X. S. Xu, and Y. X. Zhang, "Influence of inlet radius on Stokes flow in a circular tube via the Hamiltonian systematic method," Physics of Fluids, vol. 21, no. 10, Article ID 103602, 2009.

[15] J.-S. Wang and Q.-H. Qin, "Symplectic model for piezoelectric wedges and its application in analysis of electroelastic singularities," Philosophical Magazine, vol. 87, no. 2, pp. 225-251, 2007.

[16] L. Zhao and W. Q. Chen, "Symplectic analysis of plane problems of functionally graded piezoelectric materials," Mechanics of Materials, vol. 41, no. 12, pp. 1330-1339, 2009.

[17] L. Zhao and W.-Q. Chen, "On numerical calculation in symplectic approach for elasticity problems," Journal of Zhejiang University: Science A, vol. 9, no. 5, pp. 583-588, 2008. 


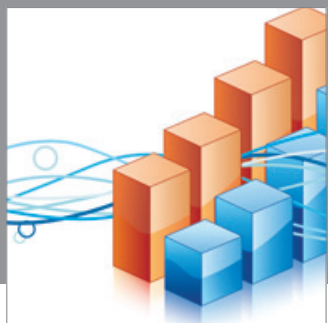

Advances in

Operations Research

mansans

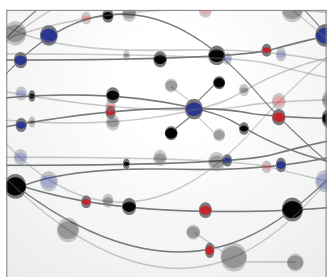

The Scientific World Journal
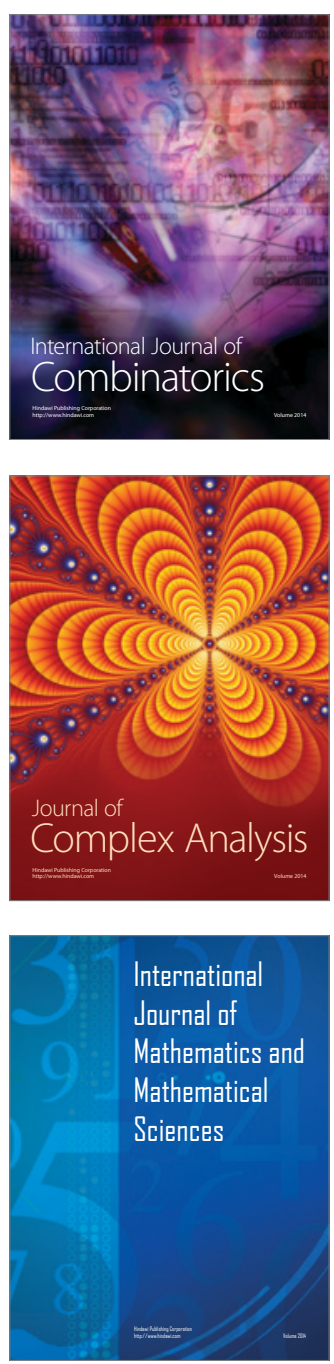
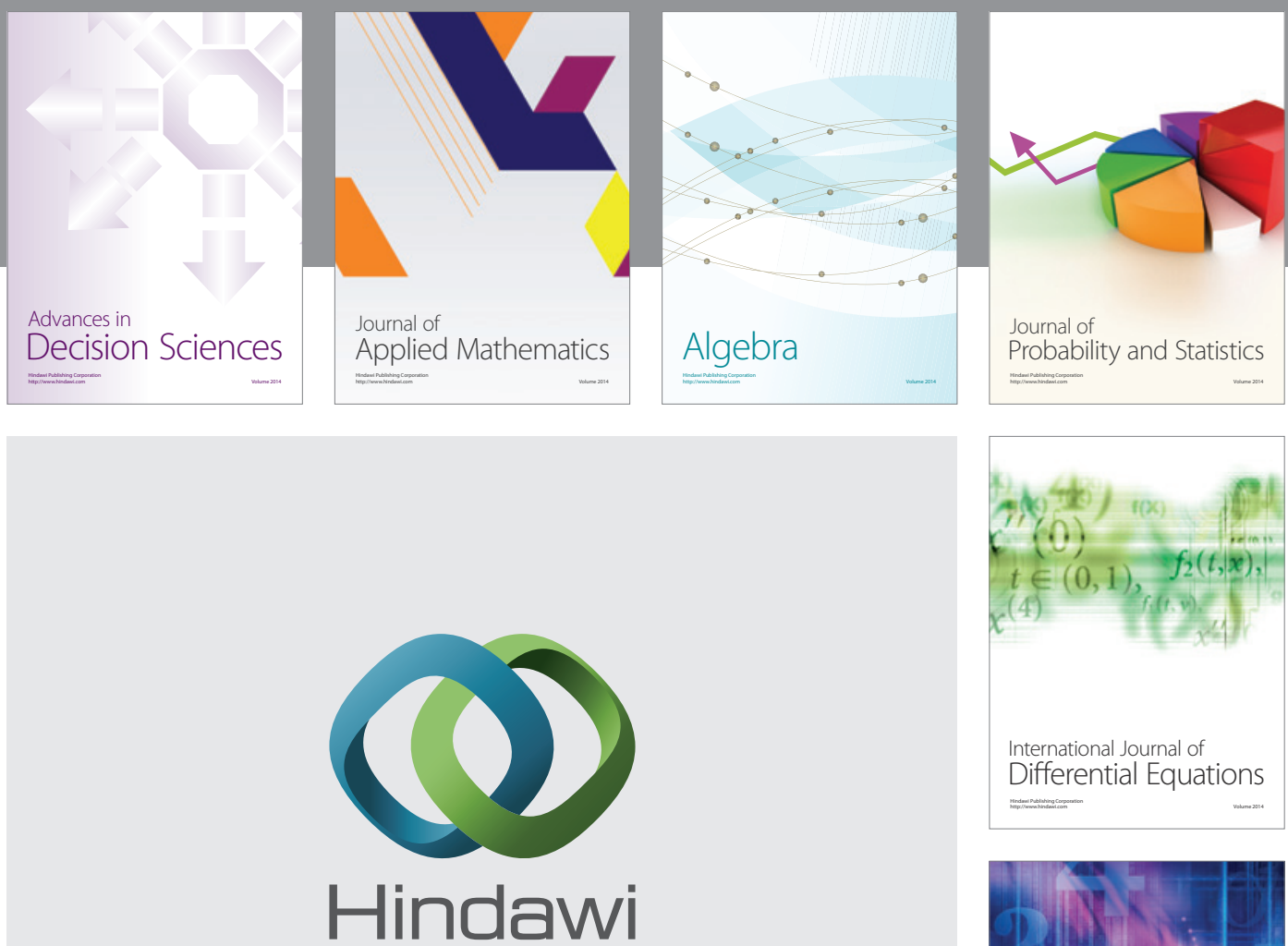

Submit your manuscripts at http://www.hindawi.com
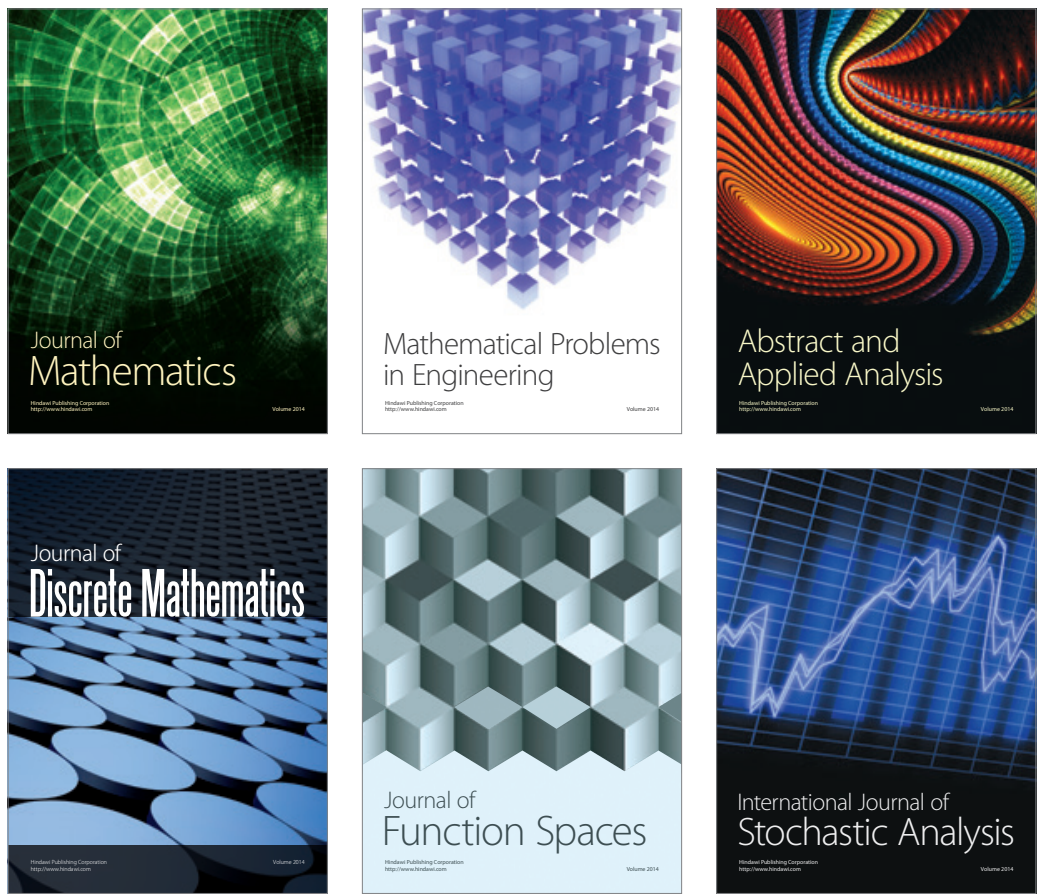

Journal of

Function Spaces

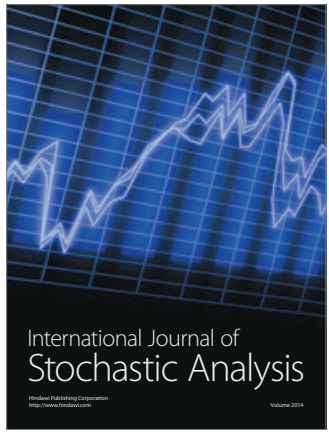

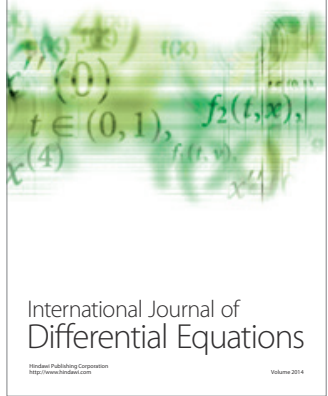
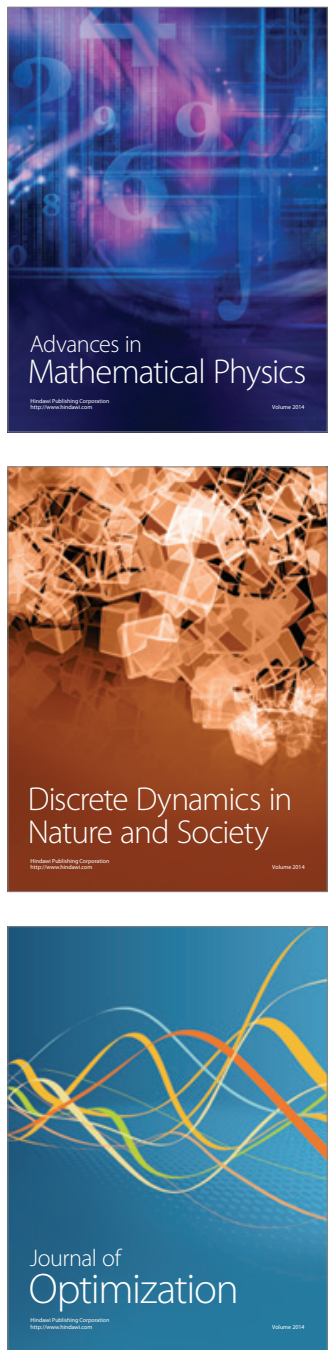